PROCEEDINGS OF THE

AMERICAN MATHEMATICAL SOCIETY

Volume 126, Number 8, August 1998, Pages 2267-2276

S $0002-9939(98) 04772-8$

\title{
PERIODIC SOLUTIONS OF THE SECOND ORDER DIFFERENTIAL EQUATIONS WITH LIPSCHITZIAN CONDITION
}

\author{
ZAIHONG WANG \\ (Communicated by Hal L. Smith)
}

\begin{abstract}
In this paper, we prove an infinity of periodic solutions to the periodically forced nonlinear Duffing equation $\ddot{x}+g(x)=p(t)$.
\end{abstract}

\section{INTRODUCTION}

Consider the Duffing equation

$$
\ddot{x}+g(x)=p(t),
$$

where $g(x), p(t) \in C(R, R)$ and $p(t)$ is periodic, whose least period is $2 \pi$. When $g(x)$ satisfies the condition

$$
0<g_{*}=\liminf _{|x| \rightarrow \infty} \frac{g(x)}{x} \leq \limsup _{|x| \rightarrow \infty} \frac{g(x)}{x}=g^{*}<\infty
$$

Eq.(1.1) is called the semi-linear Duffing equation. It is well-known that the existence problem of periodic solutions for semi-linear Eq.(1.1) is restricted by the resonant point set $\Sigma=\left\{i^{2} \mid i=0,1,2, \ldots\right\}$. In [1], D.E. Leach proved the existence and uniqueness of the $2 \pi$-periodic solution of (1.1) under Loud's condition

$$
m^{2}<\lambda \leq g^{\prime}(x) \leq \mu<(m+1)^{2},
$$

with a given integer $m \geq 0$ and two constants $\lambda$ and $\mu$. R. Reissig [2] proved the existence of periodic solutions of (1.1) under a weaker condition:

$$
m^{2}<\lambda \leq \frac{g(x)}{x} \leq \mu<(m+1)^{2}, \quad|x| \gg 1 .
$$

J. Mawhin [3] also obtained the same result. The main point of the conditions above is to exclude the resonance case. From then on, there appeared many works around the results of D.E. Leach, R. Reissig and J. Mawhin (see [4], [5], [6]).

It was T. Ding who first studied the existence problem of periodic solutions for Eq.(1.1) when $g(x)$ crosses the resonant points. Assume the following conditions hold:

Received by the editors December 10, 1996.

1991 Mathematics Subject Classification. Primary 34C25; Secondary 34B15.

Key words and phrases. Periodic solution, Poincaré-Birkhoff twist theorem.

This project was supported by NSF of China. 
$\left(g_{1}\right)$ : Let $g(x) \in C^{1}(R, R)$ and let $K$ be a positive constant such that

$$
\left|g^{\prime}(x)\right| \leq K, \quad x \in R .
$$

$\left(g_{2}\right)$ : There exist two constants $A_{0}>0$ and $M_{0}>0$ such that

$$
\frac{g(x)}{x} \geq A_{0}, \quad|x| \geq M_{0} .
$$

$\left(\tau_{0}\right)$ : There exist a constant $\sigma>0$, an integer $m>0$, and two sequences $\left\{a_{k}\right\}$ and $\left\{b_{k}\right\}(k \in N)$, such that $a_{k} \rightarrow \infty$ and $b_{k} \rightarrow \infty$ as $k \rightarrow \infty$; and moreover

$$
\tau\left(a_{k}\right)<\frac{2 \pi}{m}-\sigma, \quad \tau\left(b_{k}\right)>\frac{2 \pi}{m}+\sigma,
$$

where $\tau(e)$ denotes the least positive period of the solution $x(t)(x(0)=0, \dot{x}(0)=$ $\sqrt{2 e}$ ) for the equation $\ddot{x}+g(x)=0$.

By using a generalized form of the Poincaré-Birkhoff twist theorem, T. Ding [6] proved that Eq.(1.1) has infinitely many $2 \pi$-periodic solutions. Lately, T. Ding, R. Iannaci and F. Zanolin [7] further generalized the condition $\left(\tau_{0}\right)$ to the following condition:

$$
\left(\tau_{1}\right) \quad \Delta \tau=\limsup _{e \rightarrow \infty} \tau(e)-\liminf _{e \rightarrow \infty} \tau(e)>0 .
$$

D. Qian [8] improved the results in [6], [7] by dropping the condition $\left(g_{1}\right)$ and assuming, for $G(x)=\int_{0}^{x} g(s) d s$,

$$
\limsup _{|x| \rightarrow \infty} \frac{G(x)}{g^{2}(x)}<\infty
$$

This hypothesis is always satisfied if condition (1.2) holds. Hao Dunyuan and Ma Shiwang [9] extended the results in [6], [7] by improving conditions on the timemapping.

In the present paper, we study the existence of $2 \pi$-periodic solutions for Eq.(1.1) under conditions $\left(\tau_{0}\right)$ and

$\left(H_{1}\right): \lim _{|x| \rightarrow \infty} \operatorname{sgn}(x) g(x)=\infty$,

$\left(H_{2}\right): g(x)$ satisfies the globally lipschitzian condition. That is, there exists a positive constant $a$ such that $|g(x)-g(y)| \leq a|x-y|$.

The main difference between conditions $\left(g_{1}\right),\left(g_{2}\right)$ and $\left(H_{1}\right),\left(H_{2}\right)$ lies in that the former implies the semi-linear condition (1.2), but the latter doesn't. By developing an idea in [6], we obtain the following

Theorem 1.1. Assume that conditions $\left(H_{1}\right),\left(H_{2}\right)$ and $\left(\tau_{0}\right)$ hold. Then Eq.(1.1) possesses infinitely many $2 \pi$-periodic solutions $\left\{x_{k}(t)\right\}_{k=1}^{\infty}$ which satisfy

$$
\lim _{k \rightarrow \infty}\left(\min _{t \in R}\left(\left|x_{k}(t)\right|+\left|\dot{x}_{k}(t)\right|\right)\right)=\infty .
$$

Theorem 1.2. Assume that conditions $\left(H_{1}\right),\left(H_{2}\right)$ and $\left(\tau_{1}\right)$ hold. Then Eq.(1.1) possesses at least one $2 \pi$-periodic solution. Moreover, there exists a positive integer $n_{0}$ such that, for any integer $n \geq n_{0}$, Eq.(1.1) possesses a sequence of infinitely many periodic solutions $\left\{x_{n, k}(t)\right\}_{k=1}^{\infty}$ of minimal period $2 n \pi$ which satisfy

$$
\lim _{k \rightarrow \infty}\left(\min _{t \in R}\left(\left|x_{n, k}(t)\right|+\left|\dot{x}_{n, k}(t)\right|\right)\right)=\infty .
$$

In section 4, we construct an example for an application of the above theorems. This example also shows that Theorem 1.1 and Theorem 1.2 are not contained in the results of the previously quoted articles. 


\section{Several Lemmas}

At first, we consider the auxiliary autonomous equation

$$
\ddot{x}+g(x)=0
$$

or, its equivalent system

$$
\dot{u}=w, \quad \dot{w}=-g(u) .
$$

The orbits $\Gamma_{e}$ of the autonomous system (2.2) are curves determined by the equation

$$
\frac{1}{2} w^{2}+G(u)=e
$$

where $e$ is an arbitrary constant.

Then, we can easily prove the following:

Lemma 2.1. If $\left(H_{1}\right)$ holds, then there is a constant $e_{0}>0$, such that, for each $e \geq e_{0}, \Gamma_{e}$ is a closed curve which is star-shaped with respect to the origin $O$.

It follows from Lemma 2.1 that each curve $\Gamma_{e}\left(e \geq e_{0}\right)$ intersects the $u$-axis at two points $(d(e), 0)$ and $(c(e), 0)$, where $d(e)<0$ and $c(e)>0$ are uniquely determined by the formula

$$
G(d(e))=G(c(e))=e .
$$

Let $(u(t), w(t))$ be any solution of $(2.2)$ whose orbit is $\Gamma_{e}\left(e \geq e_{0}\right)$. Clearly, this solution is periodic. Let $\tau(e)$ denote the least positive period of this solution. It follows from (2.2) and (2.3) that

$$
\tau(e)=\sqrt{2} \int_{d(e)}^{c(e)} \frac{d u}{\sqrt{e-G(u)}} .
$$

By the definition, it follows that $\tau(e)$ is continuous for $e \geq e_{0}$.

Now we perform some phase-plane analysis for Eq.(1.1). First of all, we write the Duffing equation (1.1) in the equivalent system:

$$
\dot{x}=y, \quad \dot{y}=-g(x)+p(t) .
$$

Let $\left(x\left(t, x_{0}, y_{0}\right), y\left(t, x_{0}, y_{0}\right)\right)$ be the solution of the system (2.4) through the initial point $(x(0), y(0))=\left(x_{0}, y_{0}\right)$. It is not hard to show that every solution satisfying the initial value problem exists uniquely on the whole $t$-axis under conditions $\left(H_{1}\right)$ and $\left(H_{2}\right)$. Then the Poincaré mapping $P: R^{2} \mapsto R^{2}$ is well defined by

$$
\left(x_{0}, y_{0}\right) \mapsto\left(x\left(2 \pi, x_{0}, y_{0}\right), y\left(2 \pi, x_{0}, y_{0}\right)\right) .
$$

It is well known that $P$ is an area-preserving homeomorphism.

By applying the transformation $x(t)=r(t) \cos \theta(t), y(t)=r(t) \sin \theta(t)$ to the system (2.4), we get the equations for $r(t)$ and $\theta(t)$,

$$
\begin{aligned}
& \dot{r}=r \sin \theta \cos \theta-g(r \cos \theta) \sin \theta+p(t) \sin \theta, \\
& \dot{\theta}=-\sin ^{2} \theta-\frac{1}{r}(g(r \cos \theta) \cos \theta-p(t) \cos \theta) .
\end{aligned}
$$

Let $\left(r\left(t, r_{0}, \theta_{0}\right), \theta\left(t, r_{0}, \theta_{0}\right)\right)$ be the solution of equations (2.5) through the initial point $(r(0), \theta(0))=\left(r_{0}, \theta_{0}\right)$. Then the mapping $P$ can also be written in the polar coordinate form

$$
r^{*}=r\left(2 \pi, r_{0}, \theta_{0}\right), \quad \theta^{*}=\theta\left(2 \pi, r_{0}, \theta_{0}\right)+2 l \pi,
$$


where $l$ is an arbitrary integer. It can be easily seen that if $\left(r_{0}, \theta_{0}\right)$ is such that

$$
r\left(t, r_{0}, \theta_{0}\right)>0, \quad t \in[0,2 \pi],
$$

then $\theta\left(2 \pi, r_{0}, \theta_{0}\right)$ is well defined and continuous in $\left(r_{0}, \theta_{0}\right)$, and moreover,

$$
\theta\left(2 \pi, r_{0}, \theta_{0}+2 \pi\right)=\theta\left(2 \pi, r_{0}, \theta_{0}\right)+2 \pi \text {. }
$$

Next, we take the transformation $u(t)=\rho(t) \cos \varphi(t), w(t)=\rho(t) \sin \varphi(t)$ to the system (2.2). The resulting equations for $\rho(t)$ and $\varphi(t)$ are

$$
\begin{aligned}
& \dot{\rho}=\rho \cos \varphi \sin \varphi-g(\rho \cos \varphi) \sin \varphi, \\
& \dot{\varphi}=-\sin ^{2} \varphi-\frac{1}{\rho} g(\rho \cos \varphi) \cos \varphi .
\end{aligned}
$$

Let $\left(\rho\left(t, \rho_{0}, \varphi_{0}\right), \varphi\left(t, \rho_{0}, \varphi_{0}\right)\right)$ be the solution of equations (2.6) through the initial point $(\rho(0), \varphi(0))=\left(\rho_{0}, \varphi_{0}\right)$. Using $(2.5)$ and (2.6), we can easily prove the following

Lemma 2.2. If $\left(H_{1}\right)$ and $\left(H_{2}\right)$ hold, then there exist positive constants $\alpha>1$ and $\Lambda_{0}>1$ such that

(1) $\rho_{0} / \alpha \leq \rho(t) \leq \alpha \rho_{0}$ and $\dot{\varphi}(t)<0, \quad$ for $t \in[0,2 \pi]$ and $\rho_{0} \geq \Lambda_{0}$.

(2) $r_{0} / \alpha \leq r(t) \leq \alpha r_{0}$ and $\dot{\theta}(t)<0$, for $t \in[0,2 \pi]$ and $r_{0} \geq \Lambda_{0}$.

Lemma 2.3. Assume that conditions $\left(H_{1}\right),\left(H_{2}\right)$ and $\left(\tau_{0}\right)$ hold and let $\Phi\left(\rho_{0}, \varphi_{0}\right)$ $=\varphi\left(2 \pi, \rho_{0}, \varphi_{0}\right)-\varphi_{0}$. Then, for any constant $c>0$, there exists a constant $\Lambda>0$ such that

$$
\begin{aligned}
& \Phi\left(\rho_{0}, \varphi_{0}\right) \leq-2 m \pi-\frac{c}{\rho_{0}}, \quad\left(\rho_{0} \cos \varphi_{0}, \rho_{0} \sin \varphi_{0}\right) \in \Gamma_{a_{k}}, a_{k} \geq \Lambda, \\
& \Phi\left(\rho_{0}, \varphi_{0}\right) \geq-2 m \pi+\frac{c}{\rho_{0}}, \quad\left(\rho_{0} \cos \varphi_{0}, \rho_{0} \sin \varphi_{0}\right) \in \Gamma_{b_{k}}, b_{k} \geq \Lambda .
\end{aligned}
$$

Proof. It follows from condition $\left(H_{1}\right)$ that there exists a positive constant $\beta$ such that, if $|x| \geq \beta$, then

$$
\operatorname{sgn}(x) g(x) \geq \frac{2 c \alpha}{m \sigma}
$$

where $\alpha$ is given in Lemma 2.2 and $\sigma$ is given in condition $\left(\tau_{0}\right)$. Set

$$
E=\max _{-\beta \leq x \leq \beta}|g(x)| .
$$

Assume curve $\Gamma_{e}$ intersects the lines $x=\beta$ and $x=-\beta$ at points $\left(\beta, y_{1}\right),\left(\beta, y_{2}\right)$ and $\left(-\beta, y_{3}\right),\left(-\beta, y_{4}\right)$, respectively. Obviously, we can take $e_{0}^{*}$ large enough so that, for $e \geq e_{0}^{*}$, we have

$$
\min \left\{\left|\arctan \frac{y_{i}}{\beta}\right|: i=1,2,3,4\right\} \geq \frac{\pi}{3},
$$

and

$$
\rho_{0} \geq \Lambda_{0}, \quad 0<\frac{\alpha E}{\rho_{0}} \leq \frac{1}{4}, \quad \text { for } \quad\left(\rho_{0} \cos \varphi_{0}, \rho_{0} \sin \varphi_{0}\right) \in \Gamma_{e},
$$

where $\Lambda_{0}$ is given in Lemma 2.2.

Set

$$
D_{1}=\{(x, y)|| x \mid \leq \beta\}, D_{2}=\{(x, y)|| x|\geq \beta,| y|\geq \sqrt{3}| x \mid\}, D_{3}=R^{2}-D_{1} \cup D_{2} .
$$


If $(\rho(t), \varphi(t)) \in \Gamma_{e}, e \geq e_{0}^{*}$ and $(\rho(t), \varphi(t)) \in D_{1}$, then

$$
\dot{\varphi}\left(t, \rho_{0}, \varphi_{0}\right) \leq-\sin ^{2}\left(\frac{\pi}{3}\right)+\frac{1}{4}=-\frac{1}{2} .
$$

If $(\rho(t), \varphi(t)) \in \Gamma_{e}, e \geq e_{0}^{*}$ and $(\rho(t), \varphi(t)) \in D_{2}$, then

$$
\dot{\varphi}\left(t, \rho_{0}, \varphi_{0}\right) \leq-\sin ^{2}\left(\frac{\pi}{3}\right)-\frac{1}{\rho(t)} g(\rho(t) \cos \varphi(t)) \cos \varphi(t) \leq-\frac{3}{4} .
$$

If $(\rho(t), \varphi(t)) \in \Gamma_{e}, e \geq e_{0}^{*}$ and $(\rho(t), \varphi(t)) \in D_{3}$, then

$$
\dot{\varphi}\left(t, \rho_{0}, \varphi_{0}\right) \leq-\frac{1}{\rho(t)} g(\rho(t) \cos \varphi(t)) \cos \varphi(t) \leq-\frac{c}{m \sigma \rho_{0}} .
$$

It follows from $(2.9),(2.10)$ and (2.11) that

$$
\dot{\varphi}\left(t, \rho_{0}, \varphi_{0}\right) \leq-\frac{c}{m \sigma \rho_{0}},
$$

for $\rho_{0}$ sufficiently large. Assume $\left(u\left(t, u_{0}, w_{0}\right), w\left(t, u_{0}, w_{0}\right)\right)$ is any solution of $(2.2)$ whose orbit is $\Gamma_{a_{k}}\left(a_{k} \geq e_{0}\right)$. Since the solution $\left(u\left(t, u_{0}, w_{0}\right), w\left(t, u_{0}, w_{0}\right)\right)$ has the least period $\tau\left(a_{k}\right)$, we see the time in which $\varphi$ has a decrement $2 \pi$ is just $\tau\left(a_{k}\right)$. Write

$$
\Phi\left(\rho_{0}, \varphi_{0}\right)=\varphi\left(2 \pi, \rho_{0}, \varphi_{0}\right)-\varphi_{0}=-2 l \pi-\eta,
$$

where $l \geq 0$ is an integer, and $0 \leq \eta<2 \pi$. Let $t_{\eta}$ denote the time in which $\varphi(t)$ decreases from $\varphi_{0}-2 l \pi$ to $\varphi_{0}-2 l \pi-\eta$. Then we have

$$
l \tau\left(a_{k}\right)+t_{\eta}=2 \pi .
$$

Since $0 \leq t_{\eta}<\tau\left(a_{k}\right)$, we obtain

$$
2 \pi=l \tau\left(a_{k}\right)+t_{\eta}<(l+1) \tau\left(a_{k}\right) \leq(l+1)\left(\frac{2 \pi}{m}-\sigma\right) .
$$

It follows from (2.13) that $l \geq m$. If $l \geq m+1$, we have

$$
\Phi\left(\rho_{0}, \varphi_{0}\right) \leq-2 l \pi \leq-2(m+1) \pi .
$$

Now, assume $l=m$. Then we have

$$
t_{\eta}=2 \pi-m \tau\left(a_{k}\right) \geq 2 \pi-m\left(\frac{2 \pi}{m}-\sigma\right)=m \sigma .
$$

Therefore, we have

$$
-\eta=\int_{l \tau\left(a_{k}\right)}^{l \tau\left(a_{k}\right)+t_{\eta}} \dot{\varphi}\left(t, \rho_{0}, \varphi_{0}\right) d t \leq-\frac{c}{\rho_{0}} .
$$

Thus,

$$
\Phi\left(\rho_{0}, \varphi_{0}\right) \leq-2 l \pi-\eta \leq-2 m \pi-\frac{c}{\rho_{0}} .
$$

Combining (2.14) and (2.16) yields

$$
\Phi\left(\rho_{0}, \varphi_{0}\right) \leq-2 m \pi-\frac{c}{\rho_{0}}, \quad\left(\rho_{0} \cos \varphi_{0}, \rho_{0} \sin \varphi_{0}\right) \in \Gamma_{a_{k}},
$$

with $k$ large enough. The second inequality of Lemma 2.3 can be proved in a similar way. The proof of Lemma 2.3 is then completed. 
Lemma 2.4. Assume that conditions $\left(H_{1}\right),\left(H_{2}\right)$ hold and let

$$
\Phi\left(r_{0}, \theta_{0}\right)=\varphi\left(2 \pi, r_{0}, \theta_{0}\right)-\theta_{0}, \quad \Theta\left(r_{0}, \theta_{0}\right)=\theta\left(2 \pi, r_{0}, \theta_{0}\right)-\theta_{0} .
$$

Then there exist two positive constants $c_{0}$ and $\Gamma_{0}$ such that

$$
\left|\Phi\left(r_{0}, \theta_{0}\right)-\Theta\left(r_{0}, \theta_{0}\right)\right| \leq \frac{c_{0}}{r_{0}}, \quad \text { for } \quad r_{0} \geq \Gamma_{0} .
$$

Proof. The proof follows from arguments in [6]. Let $\left(u\left(t, x_{0}, y_{0}\right), w\left(t, x_{0}, y_{0}\right)\right)$ be the solution of (2.2) through the initial point $(u(0), w(0))=\left(x_{0}, y_{0}\right)$. Let

$$
\begin{aligned}
& s(t)=s\left(t, x_{0}, y_{0}\right)=x\left(t, x_{0}, y_{0}\right)-u\left(t, x_{0}, y_{0}\right), \\
& v(t)=v\left(t, x_{0}, y_{0}\right)=y\left(t, x_{0}, y_{0}\right)-w\left(t, x_{0}, y_{0}\right) .
\end{aligned}
$$

Then we have

$$
\begin{aligned}
& \frac{d s(t)}{d t}=v(t), \\
& \frac{d v(t)}{d t}=p(t)+g\left(u\left(t, x_{0}, y_{0}\right)\right)-g\left(x\left(t, x_{0}, y_{0}\right)\right) .
\end{aligned}
$$

Let $\eta(t)=\left(s^{2}(t)+v^{2}(t)\right)^{\frac{1}{2}}$. Then we have

$$
\eta(t) \frac{d \eta(t)}{d t}=s(t) v(t)+p(t) v(t)+\left[g\left(u\left(t, x_{0}, y_{0}\right)\right)-g\left(x\left(t, x_{0}, y_{0}\right)\right)\right] v(t) .
$$

It follows from $\left(\mathrm{H}_{2}\right)$ that

$$
\left|\eta(t) \frac{d \eta(t)}{d t}\right| \leq|p(t) v(t)|+(1+a)|s(t) v(t)|
$$

Furthermore,

$$
\left|\frac{d \eta(t)}{d t}\right| \leq \frac{1}{2}(1+a) \eta(t)+B
$$

where $B=\max _{t \in[0,2 \pi]}|p(t)|$. The differential inequality (2.17) together with $\eta(0)=$ 0 yields

$$
\eta(t) \leq \frac{2 B}{1+a}\left[e^{(1+a) \pi}-1\right] \equiv H_{0},
$$

for $t \in[0,2 \pi]$. Write $\psi(t)=\psi\left(t, r_{0}, \theta_{0}\right)=\varphi\left(t, r_{0}, \theta_{0}\right)-\theta\left(t, r_{0}, \theta_{0}\right)$. It is clear that if $|\psi(t)|<\pi$, then $\psi(t)$ is just the angle between the vectors $(x(t), y(t))$ and $(u(t), w(t))$. Therefore, we have

$$
\cos \psi(t)=\frac{r^{2}(t)+\rho^{2}(t)-\eta^{2}(t)}{2 r(t) \rho(t)} \geq 1-\frac{H_{0}^{2}}{2 r(t) \rho(t)}, \quad t \in[0,2 \pi] .
$$

On the other hand, we have $\rho(t) \geq r(t)-H_{0}$. It follows from Lemma 2.2 that, for $r_{0}$ sufficiently large, we have

$$
\frac{1}{\alpha^{2}}-\frac{H_{0}}{\alpha r_{0}}>\frac{1}{4 \alpha^{2}}
$$

and

$$
r(t)-H_{0}>0, \quad \text { for } \quad t \in[0,2 \pi] .
$$

Therefore,

$$
1-\cos \psi(t) \leq \frac{H_{0}^{2}}{2 r(t)\left(r(t)-H_{0}\right)}, \quad t \in[0,2 \pi]
$$


This results in

$$
\sin ^{2}\left(\frac{1}{2} \psi(t)\right) \leq \frac{H_{0}^{2}}{4 r(t)\left(r(t)-H_{0}\right)}, \quad \text { for } \quad t \in[0,2 \pi] .
$$

It follows from Lemma 2.2 and inequality (2.19) that

$$
\left|\sin \left(\frac{1}{2} \psi(t)\right)\right| \leq \frac{H_{0}}{2 r_{0} \sqrt{\frac{1}{\alpha^{2}}-\frac{H_{0}}{\alpha r_{0}}}} .
$$

Since $\psi(0)=0$ and $\psi(t)$ varies continuously as $t$ increases from 0 to $2 \pi$, we can see from $(2.20)$ that

$$
|\psi(t)| \leq 4\left|\sin \left(\frac{1}{2} \psi(t)\right)\right|, \quad \text { for } \quad t \in[0,2 \pi],
$$

for $r_{0}$ large enough.

Combining (2.18), (2.20) and (2.21) yields

$$
|\psi(t)| \leq \frac{4 \alpha H_{0}}{r_{0}}, \quad \text { for } \quad t \in[0,2 \pi] .
$$

Write $c_{0}=4 \alpha H_{0}$. Then we have, in particular,

$$
\left|\Phi\left(r_{0}, \theta_{0}\right)-\Theta\left(r_{0}, \theta_{0}\right)\right| \leq \frac{c_{0}}{r_{0}},
$$

for $r_{0}$ large enough.

\section{The PROOF OF THE MAIN RESUlts}

At first, we restate a generalized form of the Poincaré-Birkhoff fixed point theorem.

Let $D$ denote an annular region in the $(x, y)$-plane. The boundary of $D$ consists of two simple closed curves: the inner boundary curve $C_{1}$ and the outer boundary curve $C_{2}$. Let $D_{1}$ denote the simple connected open set bounded by $C_{1}$. Consider an area-preserving mapping $T: R^{2} \mapsto R^{2}$. Suppose that $T(D) \subset R^{2}-\{O\}$, where $O$ is the origin. Let $\left(r_{0}, \theta_{0}\right)$ be the polar coordinate of $\left(x_{0}, y_{0}\right)$, that is, $x_{0}=r_{0} \cos \theta_{0}, y_{0}=r_{0} \sin \theta_{0}$. Write the mapping $T$ in the form

$$
r^{*}=f\left(r_{0}, \theta_{0}\right), \quad \theta^{*}=\theta_{0}+h\left(r_{0}, \theta_{0}\right),
$$

where $f$ and $h$ are continuous in $\left(r_{0}, \theta_{0}\right)$ and $2 \pi$-periodic in $\theta_{0}$.

A generalized form of the Poincaré-Birkhoff fixed point theorem ([10]). Besides the above-mentioned assumptions, we assume that

(1): $C_{1}$ is star-shaped about the origion $O$;

(2): $O \in T\left(D_{1}\right)$;

(3):

$$
\begin{array}{ll}
h\left(r_{0}, \theta_{0}\right)>0(<0), & \left(r_{0} \cos \theta_{0}, r_{0} \sin \theta_{0}\right) \in C_{1} ; \\
h\left(r_{0}, \theta_{0}\right)<0(>0), \quad\left(r_{0} \cos \theta_{0}, r_{0} \sin \theta_{0}\right) \in C_{2} .
\end{array}
$$

Then $T$ has at least two fixed points in $D$.

Now, without loss of generality, we assume $e_{0} \leq a_{k}<b_{k}$ for all $k \in N$. Then, $\Gamma_{a_{k}}$ and $\Gamma_{b_{k}}$ bound an annular region $A_{k}$. In the following, we will apply the above theorem to show that the Poincaré mapping $P$ induced by equation (1.1) has at least two fixed points in the region $A_{k}$ (with $k$ large). As a consequence, Eq.(1.1) has infinitely many $2 \pi$-periodic solutions. 
Proof of Theorem 1.1. Using the notations in Lemma 2.3 and Lemma 2.4, we consider the Poincaré mapping $\left.P\right|_{A_{k}}: A_{k} \mapsto R^{2}$. Write the mapping $P$ in the form:

$$
r^{*}=r\left(2 \pi, r_{0}, \theta_{0}\right), \quad \theta^{*}=\theta_{0}+\Theta_{1}\left(r_{0}, \theta_{0}\right),
$$

where $\Theta_{1}\left(r_{0}, \theta_{0}\right)=\Theta\left(r_{0}, \theta_{0}\right)+2 m \pi$. It follows from Lemma 2.3 that, for $k$ large enough,

$$
\begin{aligned}
& \Phi\left(r_{0}, \theta_{0}\right) \leq-2 m \pi-\frac{2 c_{0}}{r_{0}}, \quad\left(r_{0} \cos \theta_{0}, r_{0} \sin \theta_{0}\right) \in \Gamma_{a_{k}}, \\
& \Phi\left(r_{0}, \theta_{0}\right) \geq-2 m \pi+\frac{2 c_{0}}{r_{0}}, \quad\left(r_{0} \cos \theta_{0}, r_{0} \sin \theta_{0}\right) \in \Gamma_{b_{k}},
\end{aligned}
$$

where $c_{0}$ is a constant given in Lemma 2.4. Applying Lemma 2.4, we have

$$
\left|\Theta_{1}\left(r_{0}, \theta_{0}\right)-\Phi\left(r_{0}, \theta_{0}\right)-2 m \pi\right| \leq \frac{c_{0}}{r_{0}}
$$

whenever $r_{0}$ is sufficiently large. Using (2.22), (2.23), we obtain

$$
\begin{array}{ll}
\Theta_{1}\left(r_{0}, \theta_{0}\right)<0, & \left(r_{0} \cos \theta_{0}, r_{0} \sin \theta_{0}\right) \in \Gamma_{a_{k}}, \\
\Theta_{1}\left(r_{0}, \theta_{0}\right)>0, & \left(r_{0} \cos \theta_{0}, r_{0} \sin \theta_{0}\right) \in \Gamma_{b_{k}},
\end{array}
$$

whenever $k$ is sufficiently large.

Thus we have proved that the area-preserving homeomorphism $P$ is twisting on the annulus $A_{k}$. Moreover, we also have that, for $k$ sufficiently large,

$$
O \in P\left(D_{k}\right)
$$

where $D_{k} \subset R^{2}$ is a open bounded set with boundary $\Gamma_{a_{k}}$. Finally, by Lemma 2.1, $\Gamma_{a_{k}}$ is star-shaped with respect to the origin $O$ (for $k$ large), so that all the assumptions of the generalized Poincaré-Birkhoff fixed point theorem are fulfilled. Therefore, we have proved that, for each $k$ large enough, the mapping $P$ has at least two fixed points in $A_{k}$. Thus we have obtained the existence of a sequence $\left\{x_{k}(t)\right\}_{k=1}^{\infty}$ of periodic solutions of Eq.(1.1), having minimal period $2 \pi$ and such that

$$
\lim _{k \rightarrow \infty}\left(\min _{t \in R}\left(\left|x_{k}(t)\right|+\left|\dot{x}_{k}(t)\right|\right)\right)=\infty .
$$

The proof of Theorem 1.1 is thus completed.

Similarly, applying methods in [7] and methods in proving Lemma 2.3 and Lemma 2.4, we can prove Theorem 1.2.

From Theorem 1.1 and Theorem 1.2, we know that the existence problem of periodic solutions for Eq.(1.1) has a tight relation with the property of the time mapping $\tau($.$) . In case g(x)$ is odd, we can easily check conditions $\left(\tau_{0}\right)$ and $\left(\tau_{1}\right)$. Set

$$
G_{*}^{+}=\liminf _{x \rightarrow \infty} \frac{2 G(x)}{x^{2}}, \quad G_{+}^{*}=\limsup _{x \rightarrow \infty} \frac{2 G(x)}{x^{2}} .
$$

Then we can prove

Lemma $3.1([7])$. Assume $g(x)$ is odd and $G_{*}^{+}<G_{+}^{*}$. Then

$$
\left[\frac{2 \pi}{\sqrt{G_{+}^{*}}}, \frac{2 \pi}{\sqrt{G_{*}^{+}}}\right] \subset\left[\tau_{*}, \tau^{*}\right]
$$

where $\tau_{*}=\liminf _{e \rightarrow \infty} \tau(e), \tau^{*}=\limsup _{e \rightarrow \infty} \tau(e)$. 
Applying Lemma 3.1 and Theorem 1.1, we obtain the following

Corollary 3.2. Assume that $\left(H_{1}\right),\left(H_{2}\right)$ hold and let $g(x)$ be an odd function. If

$$
\exists m \in N, \quad m^{2} \in\left(G_{*}^{+}, G_{+}^{*}\right),
$$

then Eq.(1.1) has an infinite sequence $\left\{x_{j}(t)\right\}_{j=1}^{\infty}$ of $2 \pi$-periodic solutions which satisfy

$$
\lim _{j \rightarrow \infty}\left(\min _{t \in R}\left(\left|x_{j}(t)\right|+\left|\dot{x}_{j}(t)\right|\right)\right)=\infty .
$$

Remark. All the results of this section are still valid if, in place of $\left(H_{2}\right)$, the assumption

$$
\exists L_{0}, K_{0} \geq 0 \text {, such that }|g(x)-g(y)| \leq L_{0}|x-y|+K_{0}, \forall x, y \in R,
$$

holds.

\section{An example}

In this section, we give an example for the application of Theorem 1.1 obtained before.

Example. Assume $g(x)$ is an odd function and $g(0)=0$ :

$$
g(x)=(x+1)^{\frac{1}{2}}+x(1+\sin \ln x)-1, \quad \text { for } \quad x>0 .
$$

It follows from the definition that $g(x)$ satisfies condition $\left(H_{1}\right)$. By a direct calculation, we have

$$
g^{\prime}(x)=\frac{1}{2}(x+1)^{-\frac{1}{2}}+\sin \ln x+\cos \ln x+1 \quad \text { for } \quad x>0 .
$$

Obviously, $g^{\prime}(x)$ is bounded in interval $(0, \infty)$. Therefore, $g(x)$ satisfies condition $\left(H_{2}\right)$. On the other hand, it is easy to check that

$$
\liminf _{x \rightarrow \infty} \frac{g(x)}{x}=0, \quad \limsup _{x \rightarrow \infty} \frac{g(x)}{x}=2
$$

and

$$
G(x)=\frac{2}{3}(x+1)^{\frac{3}{2}}+\frac{1}{2} x^{2}+\frac{x^{2}}{5}(2 \sin \ln x-\cos \ln x)-x-\frac{2}{3}, \quad x>0 .
$$

Furthermore, we have

$$
G_{*}^{+}=\liminf _{x \rightarrow \infty} \frac{2 G(x)}{x^{2}}=1+\frac{2}{5} \liminf _{x \rightarrow \infty}(2 \sin \ln x-\cos \ln x)=1-\frac{2 \sqrt{5}}{5}
$$

and

$$
G_{+}^{*}=\limsup _{x \rightarrow \infty} \frac{2 G(x)}{x^{2}}=1+\frac{2}{5} \limsup _{x \rightarrow \infty}(2 \sin \ln x-\cos \ln x)=1+\frac{2 \sqrt{5}}{5} .
$$

Then, (4.2) and (4.3) imply that

$$
\frac{2 \pi}{\sqrt{G_{+}^{*}}}<2 \pi, \quad \frac{2 \pi}{\sqrt{G_{*}^{+}}}>4 \pi .
$$

It follows from (4.4) and Lemma 3.1 that

$$
2 \pi \in] \tau_{*}, \tau^{*}[.
$$

Applying Theorem 1.1, we obtain that Eq.(1.1) possesses infinitely many $2 \pi$ periodic solutions. 
To the best of our knowledge, none of the previously known existence theorems for Eq.(1.1) can be applied for a nonlinearity like $g(x)$ in (4.1).

\section{ACKNOWLEDGMENTS}

The author is indebted to Professor F. Zanolin for suggesting the problem and to Professor T. Ding for valuable discussions. The author is also indebted to the referee for good advice.

\section{REFERENCES}

[1] D.E. Leach, On poincaré's perturbation theorem and a theorem of W.S.Loud, J. Diff. Eqs. 7 (1970), 34-53. MR 40:4539

[2] R. Reissig, Contraction mappings and periodically perturbed nonconservative systems, Atti Accad.Naz.Lincei (Rend.Cl.Sci) 58 (1975), 696-702.

[3] J. Mawhin, Recent trends in nonlinear boundary value problems, In: Proc. 7th Int. Conf. Nonlinear Oscillations(Berlin) (G. Schmidt, ed.), Akademie-Verlag, Berlin (1977), band 1, 51-70. MR 80b:58024

[4] P. Omari and F. Zanolin, A note on nonlinear oscillations at resonance, Acta Mathemata Sinica 3 (1987), 351-361. MR 89c:34039

[5] T. Ding, Nonlinear oscillations at a point of resonance, Scientia Sinica 9A (1982), 918-931. MR 84c:34058

[6] T. Ding, An infinite class of periodic solutions of periodically perturbed Duffing equation at resonance, Proc. Amer. Math. Soc. 86 (1982), 47-54. MR 83j:34041

[7] T. Ding, R. Iannacci and F. Zanolin, Existence and multiplicity results for periodic solutions of semilinear Duffing equation, J. Diff. Eqs. 105 (1993), 364-409. MR 94g:34060

[8] D. Qian, Time-maps and Duffing equations with resonance-acrossing, Scientia Sinica (Series A) (1993), 471-479.

[9] Hao Dunyuan and Ma Shiwang, Semilinear Duffing equations crossing resonance points, J. Diff. Eqs. 133 (1997), 98-116. MR 97j:34044

[10] W.Y.Ding, A generalization of the Poincaré-Birkhoff theorem, Proc. Amer. Math. Soc. 88 (1983), 341-346. MR 84f:54053

Department of Mathematics, Capital Normal University, Beijing 100037, China 